\title{
Symmetry Extensions and Their Physical Reasons in the Kinetic and Hydrodynamic Plasma Models ${ }^{\star}$
}

\author{
Volodymyr B. TARANOV \\ Institute for Nuclear Research, 47 Nauky Ave., 03028 Kyiv, Ukraine \\ E-mail:wlatar@inet.ua \\ URL: http://www.geocities.com/vebete/mypage.html
}

Received October 31, 2007, in final form January 14, 2008; Published online January 17, 2008

Original article is available at http://www.emis.de/journals/SIGMA/2008/006/

\begin{abstract}
Characteristic examples of continuous symmetries in hydrodynamic plasma theory (partial differential equations) and in kinetic Vlasov-Maxwell models (integrodifferential equations) are considered. Possible symmetry extensions conditional and extended symmetries are discussed. Physical reasons for these symmetry extensions are clarified.
\end{abstract}

Key words: symmetry; plasma; hydrodynamic; kinetic

2000 Mathematics Subject Classification: 35A30; 35Q60; 45K05; 58J70; 22E70

\section{Introduction}

Symmetry considerations essentially help us to solve nonlinear problems of plasma physics. Among the recent publications, a survey of methods which allow us to find symmetries of integrodifferential equations of kinetic plasma theory [1] and a review of applications of symmetry methods to the hydrodynamic plasma models based on the partial differential equations [2] can be cited.

In the present paper, some characteristic examples of the symmetries are presented for various plasma theory models: electron magnetohydrodynamics, collisionless electron plasma oscillations, drift waves in plasma and multi-component collisionless plasma containing particles with equal charge to mass ratios.

In Section 2, Lie point symmetries are obtained for the partial differential equations of the electron magnetohydrodynamics by means of the standard Maple 11 program. A simple invariant solution is presented, which is in fact a background solution of a perturbation theory formalism. An additional condition is underlined which can lead to the symmetry extension.

In Section 3, continuous symmetries of the integro-differential kinetic equations of the collisionless electron plasma model are discussed. The symmetries were found in [3] by an indirect algorithm which allows us to obtain symmetries of the kinetic equations from the symmetries of an infinite set of partial differential equations for the moments of distribution functions.

In Section 4, some conditional symmetries are presented for the Hasegawa-Mima hydrodynamic model describing drift waves in a plasma. Due to these symmetries, invariant solutions are possible describing the asymptotic structure of the nonlinear waves.

In Section 5, additional symmetries for integro-differential equations of the kinetic theory of collisionless plasma containing particles with equal charge to mass ratio are considered. For example, alpha particles and deuterium ions participating in a thermonuclear reaction $\mathrm{D}^{+}+$ $\mathrm{T}^{+} \rightarrow \mathrm{He}^{++}+n+17.6 \mathrm{MeV}$ have close charge to mass ratios. By simple vector considerations

\footnotetext{
${ }^{\star}$ This paper is a contribution to the Proceedings of the Seventh International Conference "Symmetry in Nonlinear Mathematical Physics" (June 24-30, 2007, Kyiv, Ukraine). The full collection is available at http://www.emis.de/journals/SIGMA/symmetry2007.html
} 
so called extended symmetry transformations are obtained which allow us to reduce the number of equations.

Conclusions are made in Section 6.

\section{Electron magnetohydrodynamics}

Let us consider the equations of the electron magnetohydrodynamics (EMHD) [4]:

$$
\frac{\partial \Psi}{\partial t}=\nabla \times(\mathbf{v} \times \Psi), \quad \Psi=\mathbf{B}-\Delta \mathbf{B}, \quad \mathbf{v}=-\nabla \times \mathbf{B}, \quad \nabla \cdot \mathbf{B}=0,
$$

where $\Psi$ is the generalized vorticity, $\mathbf{B}$ is magnetic field strength and $\mathbf{v}$ is the hydrodynamic velocity of the electron plasma component. Nine functions, $\Psi, \mathbf{v}, \mathbf{B}$, of four independent variables $t, x, y, z$ are present in this system of partial differential equations. Nevertheless, its symmetry has been obtained by the standard Maple 11 package program.

The infinitesimal operators of Lie point symmetries of the model are as follows:

$$
\begin{aligned}
& X_{1}=\frac{\partial}{\partial t}, \quad X_{2}=\frac{\partial}{\partial x}, \quad X_{3}=\frac{\partial}{\partial y}, \quad X_{4}=\frac{\partial}{\partial z}, \\
& X_{5}=t \frac{\partial}{\partial t}-\mathbf{v} \frac{\partial}{\partial \mathbf{v}}-\mathbf{B} \frac{\partial}{\partial \mathbf{B}}-\Psi \frac{\partial}{\partial \Psi}, \\
& X_{6}=\mathbf{r} \times \frac{\partial}{\partial \mathbf{r}}+\mathbf{v} \times \frac{\partial}{\partial \mathbf{v}}+\mathbf{B} \times \frac{\partial}{\partial \mathbf{B}}+\Psi \times \frac{\partial}{\partial \Psi} .
\end{aligned}
$$

The Maple 11 program was tested previously on Korteweg-de Vries, nonlinear Schrödinger, Hasegawa-Mima and other nonlinear plasma theory models and reproduced well known symmetries. So we can expect that operators $X_{1}-X_{6}$ form a full basis of EMHD Lie point symmetry algebra.

A little doubt remains, however: maybe some additional symmetries could be obtained by the direct 'manual' use of the standard Lie algorithm.

Due to time and space homogeneity (symmetries $X_{1}$ to $X_{4}$ ) the simplest exact solution exists corresponding to the constant external magnetic field which can be directed along the $O z$ axis without the loss of generality (because of the rotation symmetry $X_{6}$ ). The field amplitude can be set as $B_{0}=1$ due to the similarity transform generated by $X_{5}$ :

$$
\mathbf{B}=\mathbf{e}_{z}, \quad \mathbf{v}=0
$$

This solution can be chosen as a background for the perturbation theory. After the substitution $\mathbf{B} \rightarrow \mathbf{e}_{z}+\mathbf{B}, \Psi \rightarrow \mathbf{e}_{z}+\Psi$ the first EMHD equation becomes

$$
\frac{\partial \Psi}{\partial t}=\frac{\partial \mathbf{v}}{\partial z}+\nabla \times(\mathbf{v} \times \Psi)
$$

but other equations remain unchanged. In this way the system of equations describing helicon waves in a plasma is usually obtained.

We can expect that EMHD model is much more symmetric. For example, nonlinear term in (2) vanishes under an additional condition:

$$
\mathbf{v} \times \Psi=\nabla F,
$$

where $F$ is arbitrary scalar function. In this way we obtain the set of linear partial differential equations and the nonlinear algebraic restriction (3). This property can be a source of rich additional symmetries. 


\section{Collision less electron plasma}

Let us consider electron collisionless plasma. In this case the Vlasov-Maxwell integro-differential system of equations holds:

$$
\begin{aligned}
& \frac{\partial f}{\partial t}+v \frac{\partial f}{\partial x}-E \frac{\partial f}{\partial v}=0, \\
& \frac{\partial E}{\partial x}=1-\int_{-\infty}^{\infty} f d v, \quad \frac{\partial E}{\partial t}=\int_{-\infty}^{\infty} v f d v,
\end{aligned}
$$

where $f(t, x, v)$ is the distribution function of the electron component of the plasma, $E(t, x)$ is the electric field strength.

We cannot find symmetries of this integro-differential model by the traditional Lie method. This can be done, however, by the indirect algorithm [3].

First, let us introduce the moments:

$$
M_{k}(t, x)=\int_{-\infty}^{\infty} v^{k} f d v, \quad k=0,1,2, \ldots
$$

and obtain an infinite set of partial differential equations for them, which is as follows:

$$
\frac{\partial M_{k}}{\partial t}+\frac{\partial M_{k+1}}{\partial x}+E k M_{k-1}=0, \quad \frac{\partial E}{\partial x}=1-M_{0}, \quad \frac{\partial E}{\partial t}=M_{1} .
$$

Then, we consider the reduced system involving only first $N+1$ equations of this set, and we can obtain by the standard Lie procedure the symmetries of this system:

$$
\begin{aligned}
& X_{1}=\frac{\partial}{\partial t}, \quad X_{2}=\frac{\partial}{\partial x}, \quad X_{3}=x \frac{\partial}{\partial x}+E \frac{\partial}{\partial E}+\sum_{k=1}^{N+1} k M_{k} \frac{\partial}{\partial M_{k}}, \\
& X_{4}=\cos (t)\left(\frac{\partial}{\partial x}+\frac{\partial}{\partial E}\right)-\sin (t) \sum_{k=1}^{N+1} k M_{k-1} \frac{\partial}{\partial M_{k}}, \\
& X_{5}=\sin (t)\left(\frac{\partial}{\partial x}+\frac{\partial}{\partial E}\right)+\cos (t) \sum_{k=1}^{N+1} k M_{k-1} \frac{\partial}{\partial M_{k}},
\end{aligned}
$$

and an additional symmetry

$$
X_{F G N}=F(t) \frac{\partial}{\partial M_{N}}+\left(G(t)-x \frac{\partial F(t)}{\partial t}\right) \frac{\partial}{\partial M_{N+1}},
$$

$F(t)$ and $G(t)$ being arbitrary functions of $t$.

The standard Maple 11 program can help us to check these results for not very large $N$ (depending on computer capacity).

Only two highest moments $M_{N}$ and $M_{N+1}$ are present in $X_{F G N}$. So only moments with numbers greater than any previously chosen are involved in the transform generated by $X_{F G N}$. In the limit $N \rightarrow \infty$ the symmetry group is generated by $X_{1}-X_{5}$.

In general, restoring kinetic symmetries from the symmetries of the corresponding set of the moments equations is a hard problem in pure mathematics. Nevertheless, for the relatively simple operators $X_{1}-X_{5}$ it was shown in [3] that in the case considered they correspond to the following infinitesimal operators of the kinetic theory:

$$
X_{1}=\frac{\partial}{\partial t}, \quad X_{2}=\frac{\partial}{\partial x}, \quad X_{3}=x \frac{\partial}{\partial x}+v \frac{\partial}{\partial v}+E \frac{\partial}{\partial E}-f \frac{\partial}{\partial f},
$$




$$
X_{4}=\cos (t)\left(\frac{\partial}{\partial x}+\frac{\partial}{\partial E}\right)-\sin (t) \frac{\partial}{\partial v}, \quad X_{5}=\sin (t)\left(\frac{\partial}{\partial x}+\frac{\partial}{\partial E}\right)+\cos (t) \frac{\partial}{\partial v} .
$$

Strictly speaking, from the definition (6) it can be readily seen that the transformations generated by the operators (8), (9) in the space of variables $t, x, v$ and $f$ lead to the transformations generated by the infinitesimals (7) in the space of variables $t, x$ and $M_{k}, k=0,1,2, \ldots$

The symmetries of the same electron collisionless plasma kinetic model (4), (5) were found in [5] by the direct algorithm, i.e. without using of an infinite set of moments equations. The same generators (8), (9) were obtained.

\section{Hasegawa-Mima model}

Let us consider an inhomogeneous plasma slab in the external homogeneous magnetic field. Electrons, unlike ions, are magnetized, smoothing an electrostatic potential $\Phi$ along the magnetic field lines. In this case, Hasegawa-Mima model equations hold [6]:

$$
\frac{\partial \Psi}{\partial t}+J(\Phi, \Psi)=\frac{\partial \Phi}{\partial y}, \quad \Psi=\Phi-\Delta_{\perp} \Phi
$$

where $\Psi$ is the generalized vorticity,

$$
J(F, G) \equiv \frac{\partial F}{\partial x} \frac{\partial G}{\partial y}-\frac{\partial G}{\partial x} \frac{\partial F}{\partial y}, \quad \Delta_{\perp} \equiv \frac{\partial^{2}}{\partial x^{2}}+\frac{\partial^{2}}{\partial y^{2}} .
$$

The simultaneous presence of the $\Phi$ and $\Delta_{\perp} \Phi$ in the second equation of (10) leads to the symmetry reduction and, as a consequence, to the absence of self-similar solutions [7].

Nevertheless, self-similar solutions can exist as a consequence of conditional symmetries [8]. The notion of conditional symmetry is discussed and large bibliography is presented in a recent paper [9].

For example, if an additional condition

$$
\frac{\partial^{2} \Phi}{\partial x^{2}}=0
$$

is fulfilled, the term $\Delta_{\perp} \Phi$ is reduced to $\frac{\partial^{2} \Phi}{\partial y^{2}}$, which leads to the symmetry extension. In this way we obtain more symmetric, but still nonlinear, set of equations, which admits the following similarity transform:

$$
X_{1}=x \frac{\partial}{\partial x}+\Phi \frac{\partial}{\partial \Phi}+\Psi \frac{\partial}{\partial \Psi},
$$

which allows us to try solutions in the form:

$$
\Phi=x F(t, y), \quad \Psi=x G(t, y) .
$$

$G=-1$ is a trivial solution which is not interesting for physics. So only the simple nonlinear exact solution with $G \neq-1$ important for physics as a nonlinear wave asymptotic, will be considered below. More complicated solutions can be presented and discussed in future papers.

For the functions $F(t, y)$ and $G(t, y)$ we obtain the equations

$$
\frac{\partial(1 /(G+1))}{\partial t}+\frac{\partial(F /(G+1))}{\partial y}=0, \quad G=F-\frac{\partial^{2} F}{\partial y^{2}} .
$$

Equations (12) have a particular solution [8]:

$$
F=\alpha(1+\beta \cos ((\omega+\delta \omega) t+q y)),
$$


so that

$$
\Phi=\alpha x(1+\beta \cos ((\omega+\delta \omega) t+q y))
$$

where $\alpha$ is arbitrary constant amplitude, constant factor $\beta$ determines the relative weight of the zonal flow and the monochromatic wave, the frequency is $\omega=q /\left(1+q^{2}\right)$ and the frequency shift is $\delta \omega=-\alpha q^{3} /\left(1+q^{2}\right)$.

Another class of conditionally symmetric invariant solutions became possible under the following condition:

$$
\frac{\partial^{2} \Phi}{\partial y^{2}}=0
$$

In this case the following similarity transform appears as a conditional symmetry:

$$
X_{2}=t \frac{\partial}{\partial t}+y \frac{\partial}{\partial y}
$$

and another class of invariant solutions is possible

$$
\Phi=F(x) \frac{y}{t}+G(x), \quad \Psi=\left(F(x)-F^{\prime \prime}(x)\right) \frac{y}{t}+G(x)-G^{\prime \prime}(x) .
$$

Pure self similar solutions $G(x)=0$ are absent in this case, but there exist solutions corresponding to the arbitrary shear flow $F(x)=0, G(x)$ being an arbitrary function of $x$.

\section{Multi-component collisionless plasma}

Let us consider $N$-component collisionless plasma. In this case the Vlasov-Maxwell integrodifferential system of equations holds:

$$
\begin{aligned}
& \frac{\partial f_{\alpha}}{\partial t}+\mathbf{v} \frac{\partial f_{\alpha}}{\partial \mathbf{r}}+\frac{e_{\alpha}}{m_{\alpha}}\left(\mathbf{E}+\frac{1}{c}[\mathbf{v} \times \mathbf{B}]\right) \frac{\partial f_{\alpha}}{\partial \mathbf{v}}=0, \\
& \nabla \times \mathbf{E}+\frac{1}{c} \frac{\partial \mathbf{B}}{\partial t}=0, \quad \nabla \cdot \mathbf{E}=4 \pi \rho, \quad \nabla \times \mathbf{B}=\frac{1}{c} \frac{\partial \mathbf{E}}{\partial t}+\frac{4 \pi}{c} \mathbf{j}, \quad \nabla \cdot \mathbf{B}=0,
\end{aligned}
$$

where $f_{\alpha}(t, \mathbf{r}, \mathbf{v})$ is the distribution function of the $\alpha-t h$ component of the plasma, $\alpha=1, \ldots, N$. Charge and mass of particles of the $\alpha$ th component are denoted by $e_{\alpha}$ and $m_{\alpha}$, respectively. Charge and current densities have the form

$$
\rho=\sum_{\alpha=1}^{N} e_{\alpha} \int_{-\infty}^{\infty} f_{\alpha} d \mathbf{v}, \quad \mathbf{j}=\sum_{\alpha=1}^{N} e_{\alpha} \int_{-\infty}^{\infty} \mathbf{v} f_{\alpha} d \mathbf{v} .
$$

Let $\alpha=1$ and $\alpha=2$ correspond to plasma components with equal charge to mass ratio of particles, for example, alpha particles and deuterium ion components, so we assume (and it is a good approximation:

$$
\frac{e_{1}}{m_{1}}=\frac{e_{2}}{m_{2}}
$$

According to (17), these components enter the Maxwell equations only as the sum

$$
e_{1} f_{1}+e_{2} f_{2}
$$


which is in fact the distribution function of the charge density of the components $\alpha=1$ and $\alpha=2$. In addition, according to (15), the functions $f_{1}, f_{2}$ and $e_{1} f_{1}+e_{2} f_{2}$ satisfy the same Vlasov equation. So the transformation [8]

$$
f_{1}^{\prime}=f_{1}-e_{2} F\left(f_{1}, f_{2}\right), \quad f_{2}^{\prime}=f_{2}+e_{1} F\left(f_{1}, f_{2}\right),
$$

where $F\left(f_{1}, f_{2}\right)$ is an arbitrary function of its arguments, leaves the Vlasov-Maxwell equations invariant, at least if we perform in (17) the summation first and the integration later.

The transformation (19) does not preserve, in general, the positiveness of the distribution functions. Moreover, they can lead to the divergence of the moments, which can be shown, for example, by choosing $F=$ const.

Nevertheless, we can choose $F\left(f_{1}, f_{2}\right)=f_{1} / e_{2}$ to obtain the simplifying transformation

$$
f_{1}^{\prime}=0, \quad e_{2} f_{2}^{\prime}=e_{1} f_{1}+e_{2} f_{2} .
$$

The simplifying transform (20) can also be obtained from the symmetry deduced from the symmetry of moments' equations,

$$
f_{1}^{\prime}=f_{1} \exp (a), \quad f_{2}^{\prime}=f_{2}+\frac{e_{1}}{e_{2}}(1-\exp (a)) f_{1},
$$

$a$ is arbitrary constant, in the limit $a \rightarrow-\infty$.

According to (20), we can choose the invariant (18) as a new distribution function and solve the system (15), (16) for

$$
e_{1} f_{1}+e_{2} f_{2}, \quad f_{3}, \quad \ldots, \quad f_{N}
$$

and the fields $\mathbf{E}$ and $\mathbf{B}$, so the number of equations is reduced by one.

\section{Conclusions}

Lie point symmetries of electron magnetohydrodynamics are obtained (Section 2), which include time and space homogeneity, self similarity and rotations. As a consequence of the symmetries, simple invariant solution (1) exists which is in fact the background solution which allows to explore nonlinear waves evolution by a perturbation formalism. Rich conditional symmetries are possible in this model due to additional conditions like (3).

Lie point symmetries of the integro differential Vlasov-Maxwell model for collisionless electron plasma obtained in [3] by the moments' method, i.e. from the symmetry of an infinite set of partial differential equations for the moments of the distribution functions, are presented in Section 3.

It is shown in Section 4 that hydrodynamic Hasegawa-Mima model for drift waves in magnetized plasma, which, in general, is not self similar, has exact asymptotic self similar solutions (14). It is possible due to the conditional symmetry of the model as a consequence of an additional condition (11). The physical reason for this symmetry extension is that nonlinear waves have a simpler shape near their critical points - nodes, crests etc.

In Section 5, by simple vector considerations, it is shown that multi-component collisionless plasma containing components with equal charge to mass ratio of particles is invariant under the transformation (19), if we perform the summation first and the integration later in the expressions for charge and current densities (17). This transformation, called the extended symmetry, does not preserve, in general, the global conditions of non negativity of distribution functions and existence of their moments. So, in contrast with the conditional symmetries, this symmetry extension is a consequence not of imposing additional conditions, but of neglecting 
some global constraints. Nevertheless, among the extended symmetries (19) we can find the simplifying transform (20), which allows us to reduce the number of equations by 1 .

The physical reason for the extended symmetry (19) is that particles with equal charge to mass ratios under the same initial conditions (coordinate and velocity) in a given electric and magnetic fields move along the same trajectories. The second physically important reason is that we consider very fast evolution of a plasma, faster than any collisions.

\section{References}

[1] Ibragimov N.H., Kovalev V.F., Pustovalov V.V., Symmetries of integro-differential equations: a survey of methods illustrated by the Benney equation, Nonlinear Dynam. 28 (2002), 135-165, math-ph/0109012.

[2] Cicogna G., Ceccherini F., Pegoraro F., Applications of symmetry methods to the theory of plasma physics, SIGMA 2 (2006), 017, 17 pages, math-ph/0602008.

[3] Taranov V.B., On the symmetry of one-dimensional high frequency motions of a collisionless plasma, Sov. J. Tech. Phys. 21 (1976), 720-726.

[4] Gordeev A.V., Kingsep A.S., Rudakov L.I., Electron magnetohydrodynamics, Phys. Rep. 243 (1994), 215465.

[5] Meleshko S.V., Application of group analysis in gas kinetics, in Proc. Joint ISAMM/FRD Inter-Disciplinary Workshop "Symmetry Analysis and Mathematical Modelling", 1998, 45-60.

[6] Horton W., Drift waves and transport, Rev. Modern Phys. 71 (1999), 735-778.

[7] Taranov V.B., Drift and ion-acoustic waves in magnetized plasmas, symmetries and invariant solutions, Ukrainian J. Phys. 49 (2004), 870-874.

[8] Taranov V.B., Symmetry extensions in kinetic and hydrodynamic plasma models, in Proceedinds of 13 th International Congress on Plasma Physics (2006, Kyiv), 2006, A041p, 4 pages.

[9] Cicogna G., Laino M., On the notion of conditional symmetry of differential equations, Rev. Math. Phys. 18 (2006), 1-18, math-ph/0603021. 